\title{
Land capability and suitability of some soils at North-West of Dashlut, Assiut, Egypt
}

\author{
Sayed Y. A.*, Khalafalla M. Y. \\ Soils and Water Science Department, Faculty of Agriculture, Al-Azhar University, Assiut, Egypt
}

\begin{abstract}
Twelve soil profiles representing North-West of Dashlut area, Assiut, Egypt were selected and dug, and 43 soil samples were collected from these profiles to evaluate its productive capability and suitability for growing selected crops. The soils of this area had a coarse texture grades (sand, loamy sand, and sandy loam). Most of these soils were highly saline $\left(\mathrm{EC}_{\mathrm{e}}>16 \mathrm{dS} / \mathrm{m}\right)$ and showed low values of organic matter and available NPK, some of these soils are considered as calcareous. Tow modern programs including the applied system of land evaluation (ASLE) and the microcomputer land evaluation information system (MicroLEIS) were applied to assess the capability of these soils and their suitability to grow selected crops. The results of land capability showed that the soils of the study area were poor (C4), very poor (C5), and non-agricultural (C6) using the ASLE program, while the MicroLEIS (Cervatana model) program pointed that soils had moderately (S3) and marginally (N1) capable grades. Moreover, using the ASLE program, the soils of the study area were highly suitable, suitable, moderately suitable, marginally suitable, and currently not suitable and permanently not suitable for 28 field and vegetable crops and fruits. The land suitability using MicroLEIS (Almagra model) program indicated that the soils of this area were moderately suitable, marginally suitable, and non-suitable for the selected crops due to one or more of the limiting factors. The geostatistics approach performed with ordinary kriging interpolation and semivariogram was applied to create a detailed and spatialized map for each soil parameter. Circular, JBessel, J-Bessel, and Stable geostatistical models were used to define the spatial variability of soil parameters based on RMS, MSE, and RMSSE. The main soil limitations of these soils were soil texture, soil salinity, and low soil fertility. However, these soil limitations are none permanent and can be improved through applying suited management practices.
\end{abstract}

Keywords: land capability, land suitability, MicroLEIS program, ASLE program.

*Corresponding author: Sayed Y. A.,

Copyright (c) 2021

E-mail address: yasser_soils@yahoo.com

(cc) $\mathrm{BY}-\mathrm{NC}$ 


\section{Introduction}

Land suitability indicates that the ability of a portion of land to tolerate the production of crops in a sustainable way (Bandyopadhyay et al., 2009). Land suitability evaluation provides information on the restrictions and opportunities for land use and therefore, it guides decisions on resources optimal use. Knowledge is an essential prerequisite for land use planning and development. Furthermore, such a type of analysis helps to identify the major limiting factors for agricultural production and enables decision-makers such as landuse planners, land users, and agricultural support services to develop the management of crops able to overcome such constraints, increasing productivity. Land could be categorized into spatially distributed agriculture potential zones based on the soil properties, terrain characteristics, and analyzing present land use (AbdelRahman et al., 2016). According to FAO (2006), land suitability is the classification operation of soil to use appropriately. This operation is the evaluation and gathering of specific areas of land in terms of their suitability for defined uses. Geostatistical techniques can give more dependable, useful, and efficient tools to predict soil properties in unknown and unsampled sites and to describe the spatial association of data by variogram analyses (Webster and Oliver, 2007). The kriging is the most effective and strong interpolation method used in geostatistical applications (Mevlut, 2016). De la Rosa et al. $(2004 ; 2009)$ designed the program of the Microcomputer Land Evaluation Information System (MicroLIES) package that has been considered a user-friendly agro-ecological decision support system for sustainable land use and management. The MicroLIES with a Cervatana and Almagra models forecast the general land capability or suitability for a broad series of possible agricultural uses. The program works interactively, comparing the values of the characteristics of the land unit with the generalization levels designated for each use capability class. The prediction of the general land use capability (Cervatana model) and the land suitability (Almagra model) are the results of a qualitative evaluation process or overall interpretation of the following biophysical factors such as relief, soil, climate, and current use or vegetation. Also, an Applied System for Land Evaluation (ASLE) program is proposed by Ismail and Morsi (2001) and Ismail et al. (2001) to evaluate land capability and suitability. This program calculates the final land capability index as a percentage value and the land suitability depends upon four characteristics, namely soil properties, irrigation water quality, soil fertility factors, and environmental parameters. Each factor was described as an index value to give its status in the percentage form (Marei et al., 1987; Zamil et al., 2009). Fadel and Sayed (2020) evaluated the soils of El-Qusiya area, Assiut, Egypt as one of the newly reclaimed areas using Storie index (O'GEEN, 2008). Their output data showed that these soils were fair (Grade 3) and poor (Grade 4) with slope and other soil limitation factors. MicroLIESCervatana model results showed that the land capability classes of this area were good (S2), moderate (S3), and marginal (N) with limiting factors of soil (i), erosion 
risks (r), and bioclimatic deficit (b). According to Sys and Verheye (1978) system, two land suitability classes, namely marginal suitable (S3) with severe limitations and presently not suitable (N1) were reported for this area. The land suitability of this area for growing different crops according to MicroLIESAlmagra model belonged to suitability classes of high suitable (S2), moderately suitable (S3), marginally suitable (S4), and not suitable (S5) for wheat, maize, watermelon, potato, soybean, cotton, sunflower, sugar beet, alfalfa, peach, citrus, and olive, with limitation factors of texture (t), drainage (d), carbonate (c), salinity (s), sodium saturation (a), and profile development (g). North-West of Dashlout area, Assiut, Egypt is considered one of the promising areas for agricultural expansion, due to its almost flat surface, neighbor of the residential areas, and its proximity to the main roads. So, the current study aims to assess the productive capacity of these lands and their suitability for growing various crops and define the main limitations of this study area.

\section{Materials and methods}

\subsection{Study Area}

The study area is located at North-West of Dashlut, Assiut, Egypt between latitude $27^{\circ} 34^{\prime} 34.4^{\prime \prime}$ to $27^{\circ} 39^{\prime} 12.5^{\prime \prime} \mathrm{N}$ and longitude $30^{\circ} 35^{\prime} 45.3^{\prime \prime}$ to $30^{\circ} 40^{\prime} 15.1^{\prime \prime} \mathrm{E}$. It covers an area of $43.94 \mathrm{~km}^{2}(10462$ feddans). Twelve soil profiles were chosen to represent the study area (Figure 1) to evaluate the capability and suitability of these soils for the growth of crops and define the limiting factors of this area. Forty-three soil samples were collected from these soil profiles. Locations of these soil profiles were recorded in the field with GPS guidance. Each soil profile was dug to $130-150 \mathrm{~cm}$ according to the type and nature of the soil material. The morphological description of these soil profiles was performed according to Soil Survey Staff (1993) and FAO (2006). The climate of this area was a thermic temperature regime and an aridic soil moisture regime where the mean annual temperature, rainfall, and relative humidity are $26{ }^{\circ} \mathrm{C}, 1.2 \mathrm{~mm}$, and $40 \%$, respectively.

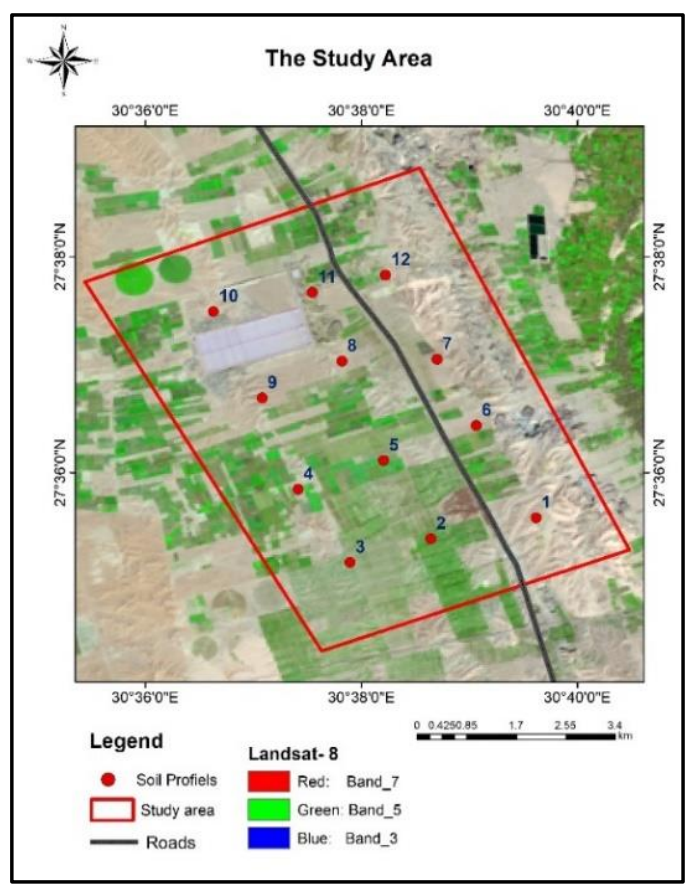

Figure (1): Location map of the study area.

\subsection{Soil Analysis}

The soil samples were air-dried, crushed, 
sieved through $2 \mathrm{~mm}$ sieve, and kept for physical and chemical analysis. In these samples, the particle-size distribution was determined by pipette method (Piper, 1950) and the saturated hydraulic conductivity was measured in the undisturbed soil cores using the constant head method (Kulte and Dirksen, 1986). The soil organic matter content (SOM) was determined by Wakley and Black method (Jackson, 1973). The soil calcium carbonate $\left(\mathrm{CaCO}_{3}\right)$ was measured by the calcimeter method according to Nelson (1982). Soil $\mathrm{pH}$ was measured in a 1:1 soil to water suspension (Mclean, 1982). The electrical conductivity of the soil saturated paste extract $\left(\mathrm{EC}_{\mathrm{e}}\right)$ was determined according to Jackson (1973). Gypsum content was determined using the acetone method (Hesse, 1998). The cation exchange capacity (CEC) was measured (Jackson, 1973). The exchangeable sodium percentage (ESP) was calculated using the values of CEC and the exchangeable sodium. Available nitrogen using Kjeldahl method (Hesse,
1998), available phosphorus using Olsen and Sommers (1982), and available potassium using flame photometer (Hesse, 1998) were determined in the collected soil samples. Landsat 8 satellite images (path 176, row 39) covering the studied area were acquired on 19-062020. The ENVI 5.1 software was implemented (ITT, 2017). The location, some soil properties, land capability, and suitability maps of the investigated soils were layout, annotated, projected, and finally produced using Arc GIS 10.2.2 software (ESRI, 2014).

\subsection{Land Capability}

The applied system of land evaluation (ASLE) program proposed by Ismail and Morsi (2001) was used to define the capability classes, C1, C2, C3, C4, C5, and C6 (Table 1). MicroLEIS (Cervatana model) internet-based program (De la Rosa et al., 2004) that has class S1, S2, S3, and $\mathrm{N}$ was also applied for land capability (Table 1).

Table (1): Land capability classes of the applied system of land evaluation (ASLE) and MicroLEIS (Cervatana model).

\begin{tabular}{|c|c|c|c|}
\hline Appli & nd Evaluation (ASLE) program & MicroL & ervatana mod \\
\hline Class & $\%$ & Class & Description \\
\hline 1 & $80-100$ (Excellent) & $\mathrm{S} 1$ & Excellent \\
\hline 2 & $60-80$ (Good) & S2 & Good \\
\hline 3 & 40-60 (Fair) & S3 & Moderate \\
\hline 4 & $20-40$ (Poor) & \multirow{3}{*}{$\mathrm{N}$} & \multirow{3}{*}{ Marginal } \\
\hline 5 & 10-20 (Very poor) & & \\
\hline 6 & $<10$ (Non-agricultural) & & \\
\hline
\end{tabular}

\subsection{Land Suitability}

The applied system of land evaluation
(ASLE) for arid and semi-arid regions (Ismail and Morsi, 2001) program based on the ratings of crop requirements 
proposed by Sys et al. (1993) was applied for different crops such as cotton, sunflower, sugar beet, wheat, barley, sugarcane, faba bean, maize, soya bean, rice, peanut, alfalfa, sorghum, vegetables, watermelon, pepper, tomato, cabbage, pea, onion, potato, date palm, fig, olive, grape, apple, pear, citric and banana (Table 2). The MicroLEIS (Almagra model) program introduced by De la Rosa et al. (2004) was used for selected crops such as alfalfa, cotton, sugar beet, maize, wheat, melon, potatoes, olive, soya bean, sunflower, citrus, and peach (Table 2).

Table (2): Land suitability grades of the applied system of land evaluation (ASLE) and MicroLEIS (Almagra model).

\begin{tabular}{|c|c|c|c|c|c|c|c|c|}
\hline \multirow{2}{*}{\multicolumn{3}{|c|}{$\begin{array}{l}\text { Applied System of Land Evaluation (ASLE) } \\
\text { program }\end{array}$}} & \multicolumn{6}{|c|}{ MicroLEIS (Almagra model) } \\
\hline & & & \multicolumn{2}{|c|}{ Suitability class } & \multicolumn{2}{|c|}{ Limitation } & \multicolumn{2}{|r|}{ Soil factor } \\
\hline S1 & $>80$ & High suitable & S1 & High suitable & 1 & None & $\mathrm{a}$ & Sodium saturation \\
\hline S3 & $30-60$ & Moderately suitable & S3 & Moderately suitable & 3 & Moderate & $\mathrm{d}$ & Drainage \\
\hline S4 & $20-30$ & Marginally suitable & S4 & Marginally suitable & 4 & Severe & $\mathrm{g}$ & Profile development \\
\hline NS1 & $10-20$ & Currently not suitable & \multirow{2}{*}{ S5 } & \multirow{2}{*}{ Not suitable } & \multirow{2}{*}{5} & \multirow{2}{*}{ Very severe } & $\mathrm{p}$ & Useful depth \\
\hline $\mathrm{NSL}$ & $<10$ & Permanentiy not suitable & & & & & $\mathrm{t}$ & Texture \\
\hline
\end{tabular}

\subsection{Geostatistical analyses}

The variability of the soil parameters was examined using the geostatistical approach. The geostatistical approach included the calculation of the experimental semivariogram and unsampled site prediction. The most advantage of geostatistics is the measuring of the spatial correlation using the semivariogram. There are different models in geostatistical analysis, including circular, spherical, tetraspherical, pentaspherical, exponenttial, gaussian, rational quadratic, hole effect, K-bessel, J-bessel, and stable. The suitability and validation of each model were examined through some parameters like mean prediction errors (MPE), root mean square prediction errors (RMSPE), mean standardized prediction errors (MSPE) that must be close to 0 and root mean square standardized prediction errors (RMSSPE) that should be close to 1 (Webster and Oliver, 2007).

\section{Results and Discussion}

\subsection{Soil characteristics}

The results show that the saturation percentage (SP) of the soil samples ranges between 18 to $28 \%$, due to the coarse texture of these soils (Table 3). Two soil texture categories are found in these soils including the coarse texture which is represented by the sand and loamy sand texture grades and the moderately coarse texture that is represented by the sandy loam texture grade according to (Sys, 1979). The hydraulic conductivity of these soil samples differs from 4.4 to 16.8 $\mathrm{cm} / \mathrm{h}$. The calcium carbonate content 
varies between 3 and $28 \%$. Some soils of the study area are considered as calcareous soils $(16-28 \%)$. The soil organic matter $(\mathrm{OM})$ is low $0.07-0.56 \%$ and the gypsum content reaches $0.74 \%$ (Figure 2).

Table (3): Some soil physical properties of the study area.

\begin{tabular}{|c|c|c|c|c|c|c|c|c|c|c|c|c|}
\hline \multirow[b]{2}{*}{ Profile No. } & \multirow[b]{2}{*}{ Deep of layer } & \multirow[b]{2}{*}{$\begin{array}{l}\text { SP } \\
(\%)\end{array}$} & \multirow[b]{2}{*}{$\begin{array}{l}\text { Gravel by } \\
\text { volume }(\%)\end{array}$} & \multicolumn{3}{|c|}{ Particle-size distribution } & \multirow[b]{2}{*}{ Texture grade } & \multirow[b]{2}{*}{$\begin{array}{c}\text { Hydraulic conductivity } \\
(\mathrm{cm} / \mathrm{h})\end{array}$} & \multirow[b]{2}{*}{$\begin{array}{l}\mathrm{CaCO}_{3} \\
(\%)\end{array}$} & \multirow[b]{2}{*}{$\begin{array}{l}\mathrm{O} . \mathrm{M} \\
(\%)\end{array}$} & \multirow[b]{2}{*}{$\begin{array}{l}\text { Gypsum } \\
(\%)\end{array}$} & \multirow[b]{2}{*}{ Land use } \\
\hline & & & & \begin{tabular}{|c|} 
Clay \\
$(\%)$
\end{tabular} & \begin{tabular}{|l} 
Silt \\
$(\%)$
\end{tabular} & $\begin{array}{c}\text { Sand } \\
(\%)\end{array}$ & & & & & & \\
\hline \multirow{4}{*}{1} & $0-20$ & 18 & 11 & 4 & 8 & 88 & Sand & 12.5 & 16 & 0.48 & 0.74 & \multirow{4}{*}{ None } \\
\hline & $20-50$ & 21 & 5 & 8 & 9 & 83 & \begin{tabular}{|l} 
Loamy sand \\
\end{tabular} & 8.8 & 13 & 0.31 & \begin{tabular}{|l|}
0.12 \\
\end{tabular} & \\
\hline & $50-90$ & 25 & 19 & 8 & 18 & 74 & \begin{tabular}{|l|} 
Gravelly sandy loam \\
\end{tabular} & 5.1 & 9 & 0.37 & \begin{tabular}{|l|}
0.53 \\
\end{tabular} & \\
\hline & $90-150$ & 23 & 25 & 5 & 15 & 80 & Gravelly loamy sand & 6.0 & 25 & 0.27 & 0.50 & \\
\hline \multirow{3}{*}{2} & $0-30$ & 20 & 20 & 2 & 6 & 92 & Gravelly sand & 10.0 & 6 & 0.34 & \begin{tabular}{|l|}
0.00 \\
\end{tabular} & \multirow{3}{*}{$\begin{array}{l}\text { Wheat, Tomato, } \\
\text { Eggplant }\end{array}$} \\
\hline & $30-70$ & 23 & 4 & 7 & 14 & 79 & Loamy sand & 6.0 & 7 & 0.37 & \begin{tabular}{|l|}
0.00 \\
\end{tabular} & \\
\hline & $70-130$ & 19 & 19 & 5 & 7 & 88 & Gravelly sand & 9.3 & 5 & 0.29 & \begin{tabular}{|l|}
0.01 \\
\end{tabular} & \\
\hline \multirow{4}{*}{3} & $0-15$ & 23 & 21 & 6 & 14 & 80 & Gravelly loamy sand & 6.7 & 11 & 0.43 & \begin{tabular}{|l|}
0.03 \\
\end{tabular} & \multirow{4}{*}{ None } \\
\hline & $15-55$ & 19 & 16 & 4 & 5 & 91 & \begin{tabular}{|l|} 
Gravelly sand \\
\end{tabular} & 11.8 & 9 & 0.26 & \begin{tabular}{|l|}
0.11 \\
\end{tabular} & \\
\hline & $55-105$ & 21 & 11 & 8 & 8 & 84 & Loamy sand & 8.2 & 14 & 0.33 & \begin{tabular}{|l|}
0.19 \\
\end{tabular} & \\
\hline & $105-150$ & 20 & 13 & 5 & 6 & 89 & Sand & 11.9 & 17 & 0.24 & \begin{tabular}{|l|}
0.02 \\
\end{tabular} & \\
\hline \multirow{4}{*}{4} & $0-20$ & 21 & 17 & 7 & 12 & 81 & Gravelly loamy sand & 7.4 & 9 & 0.49 & \begin{tabular}{|l|}
0.02 \\
\end{tabular} & \multirow{4}{*}{ None } \\
\hline & $20-70$ & 23 & 11 & 12 & 20 & 68 & \begin{tabular}{|l|} 
Sandy loam \\
\end{tabular} & 4.5 & 15 & 0.21 & \begin{tabular}{|l|}
0.01 \\
\end{tabular} & \\
\hline & $70-110$ & 20 & 19 & 4 & 7 & 89 & Gravelly sand & 9.9 & 11 & 0.19 & \begin{tabular}{|l|}
0.03 \\
\end{tabular} & \\
\hline & $110-150$ & 25 & 8 & 10 & 21 & 69 & Sandy loam & 5.9 & 16 & 0.22 & \begin{tabular}{|l|}
0.15 \\
\end{tabular} & \\
\hline \multirow{3}{*}{5} & $0-30$ & 19 & 9 & 5 & 4 & 91 & Sand & 12.9 & 10 & \begin{tabular}{|l|}
0.55 \\
\end{tabular} & \begin{tabular}{|l|}
0.00 \\
\end{tabular} & \\
\hline & $30-80$ & 24 & 17 & 10 & 18 & 72 & Gravelly sandy loam & 4.6 & 28 & 0.49 & \begin{tabular}{|l|}
0.01 \\
\end{tabular} & Wheat, Onion, Pepper \\
\hline & $80-140$ & 28 & 17 & 8 & 28 & 64 & Gravelly sandy loam & 4.5 & 26 & 0.34 & \begin{tabular}{|l|}
0.02 \\
\end{tabular} & \\
\hline & $0-25$ & 19 & 7 & 4 & 6 & 90 & \begin{tabular}{|l} 
Sand \\
\end{tabular} & 14.7 & 9 & 0.35 & \begin{tabular}{|l|}
0.01 \\
\end{tabular} & \\
\hline & $25-60$ & 24 & 11 & 8 & 20 & 72 & $\begin{array}{l}\text { Sandy loam } \\
\end{array}$ & 6.8 & 10 & 0.32 & \begin{tabular}{|l|}
0.01 \\
\end{tabular} & \\
\hline 6 & $60-90$ & 20 & 19 & 3 & 6 & 91 & \begin{tabular}{|l|} 
Gravelly sand \\
\end{tabular} & 11.4 & 4 & 0.17 & \begin{tabular}{|l|}
0.02 \\
\end{tabular} & None \\
\hline & $90-150$ & 23 & 16 & 7 & 19 & 74 & Gravelly sandy loam & 6.4 & 5 & 0.07 & \begin{tabular}{|l|}
0.01 \\
\end{tabular} & \\
\hline & $0-30$ & 20 & 11 & 6 & 4 & 90 & \begin{tabular}{|l|} 
Sand \\
\end{tabular} & 10.9 & 3 & 0.32 & \begin{tabular}{|l|}
0.00 \\
\end{tabular} & \\
\hline 7 & $30-80$ & 19 & 5 & 2 & 6 & 92 & Sand & 14.4 & 6 & \begin{tabular}{|l|}
0.43 \\
\end{tabular} & \begin{tabular}{|l|}
0.00 \\
\end{tabular} & Tomato, Onion, \\
\hline & $80-140$ & 23 & 4 & 7 & 9 & 84 & Loamy sand & 10.9 & 5 & 0.29 & \begin{tabular}{|l|}
0.01 \\
\end{tabular} & \\
\hline & $0-25$ & 27 & 7 & 11 & 21 & 68 & Sandy loam & 5.5 & 11 & 0.30 & \begin{tabular}{|l|}
0.00 \\
\end{tabular} & \\
\hline & $25-70$ & 20 & 9 & 4 & 6 & 90 & Sand & 14.4 & 9 & 0.32 & \begin{tabular}{|l|}
0.03 \\
\end{tabular} & \\
\hline 8 & $70-100$ & 19 & 22 & 3 & 7 & 90 & Gravelly sand & 8.7 & 17 & 0.27 & \begin{tabular}{|l|}
0.03 \\
\end{tabular} & None \\
\hline & $100-150$ & 21 & 11 & 5 & 16 & 79 & Loamy sand & 9.7 & 15 & 0.22 & \begin{tabular}{|l|}
0.02 \\
\end{tabular} & \\
\hline & $0-30$ & 20 & 14 & 3 & 8 & 89 & Sand & 13.5 & 13 & 0.40 & \begin{tabular}{|l|}
0.00 \\
\end{tabular} & \\
\hline 9 & $30-80$ & 24 & 8 & 9 & 21 & 70 & Sandy loam & 6.5 & 20 & 0.26 & \begin{tabular}{|l|}
0.03 \\
\end{tabular} & None \\
\hline & $80-140$ & 20 & 19 & 6 & 13 & 81 & Gravelly loamy sand & 6.8 & 10 & 0.25 & \begin{tabular}{|l|}
0.03 \\
\end{tabular} & \\
\hline & $0-30$ & 19 & 14 & 3 & 4 & 93 & \begin{tabular}{|l|} 
Sand \\
\end{tabular} & 13.6 & 14 & 0.28 & \begin{tabular}{|l|}
0.00 \\
\end{tabular} & \\
\hline 10 & $30-90$ & 23 & 7 & 7 & 33 & 60 & Sandy loam & 6.2 & 16 & 0.41 & \begin{tabular}{|l|}
0.01 \\
\end{tabular} & None \\
\hline & $90-140$ & 22 & 2 & 5 & 13 & 82 & Loamy sand & 13.0 & 11 & \begin{tabular}{|l|}
0.37 \\
\end{tabular} & \begin{tabular}{|l|}
0.11 \\
\end{tabular} & \\
\hline & $0-30$ & 19 & 9 & 4 & 7 & 89 & Sand & 13.5 & 6 & 0.38 & \begin{tabular}{|l|}
0.00 \\
\end{tabular} & \\
\hline & $30-70$ & 20 & 6 & 3 & 4 & 93 & Sand & 16.8 & 8 & 0.48 & \begin{tabular}{|l|}
0.00 \\
\end{tabular} & \\
\hline 11 & $70-120$ & 21 & 23 & 5 & 18 & 77 & Gravelly Loamy sand & 6.8 & 10 & 0.34 & \begin{tabular}{|l|}
0.00 \\
\end{tabular} & Tomato, Onion \\
\hline & $120-150$ & 21 & 3 & 8 & 9 & 83 & \begin{tabular}{|l|} 
Loamy sand \\
\end{tabular} & 9.9 & 14 & 0.29 & \begin{tabular}{|l|}
0.01 \\
\end{tabular} & \\
\hline & $0-25$ & 23 & 13 & 13 & 17 & 70 & Sandy loam & 4.4 & 11 & 0.56 & 0.02 & \\
\hline & $25-55$ & 22 & 11 & 8 & 14 & 78 & Loamy sand & 7.3 & 15 & 0.34 & \begin{tabular}{|l|}
0.01 \\
\end{tabular} & Wheat, Tomato, \\
\hline 12 & $55-110$ & 25 & 12 & 9 & 31 & 60 & Sandy loam & 4.8 & 10 & \begin{tabular}{|l|}
0.49 \\
\end{tabular} & 0.02 & Onion, Pepper \\
\hline & $110-150$ & 19 & 9 & 3 & 7 & 90 & Sand & 16.8 & 16 & 0.22 & 0.01 & \\
\hline
\end{tabular}
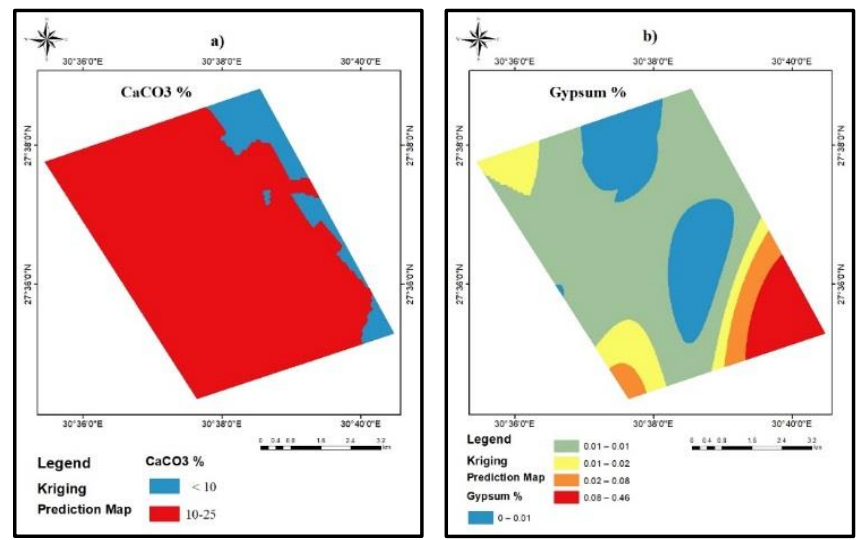

Figure (2): Spatial variability of a) the soil calcium carbonate $\left(\mathrm{CaCO}_{3}\right)$ and $\left.\mathrm{b}\right)$ gypsum contents of the study area. 
The electrical conductivity of the saturated soil paste extract $\left(\mathrm{EC}_{\mathrm{e}}\right)$ of these soils ranges widely from 3.1 to 119.1 $\mathrm{dS} / \mathrm{m}$ (Table 4). Most of these soils are highly saline that have $\mathrm{EC}_{\mathrm{e}}>16 \mathrm{dS} / \mathrm{m}$ (Figure 3). The soil $\mathrm{pH}$ of these soils varies from 7.1 to 9.4. The cation exchange capacity (CEC) is between 3 and $18 \mathrm{cmol} \mathrm{(+)} / \mathrm{kg}$. It has low values due to the prevailing coarse texture and the low colloid fraction of these soils. However, most of the study area has a soil exchange sodium percentage that is less than $15 \%$.

Table (4): Some soil chemical characteristics of the study area.

\begin{tabular}{|c|c|c|c|c|c|c|c|c|c|c|c|c|c|c|c|}
\hline \multirow[b]{2}{*}{$\begin{array}{l}\text { Profile } \\
\text { No. }\end{array}$} & \multirow[b]{2}{*}{$\begin{array}{l}\text { Deep of } \\
\text { layer }\end{array}$} & \multirow[b]{2}{*}{$\begin{array}{l}\mathrm{pH} \\
1: 1\end{array}$} & \multirow[b]{2}{*}{$\begin{array}{r}E C_{e} \\
d S / m\end{array}$} & \multicolumn{4}{|c|}{ Cations (mmol/kg) } & \multicolumn{3}{|c|}{ Anions (mmol/kg) } & \multirow[b]{2}{*}{$\begin{array}{c}\text { CEC } \\
\mathrm{cmol}(+) / \mathrm{kg})\end{array}$} & \multirow[b]{2}{*}{$\begin{array}{l}\text { ESP } \\
(\%)\end{array}$} & \multirow{2}{*}{$\begin{array}{l}\text { Available } \\
\text { nitrogen } \\
(\mathrm{mg} / \mathrm{kg})\end{array}$} & \multirow{2}{*}{$\begin{array}{c}\text { Available } \\
\text { phosphorus } \\
(\mathrm{mg} / \mathrm{kg})\end{array}$} & \multirow{2}{*}{$\begin{array}{c}\text { Available } \\
\text { potassium } \\
(\mathrm{mg} / \mathrm{kg})\end{array}$} \\
\hline & & & & $\mathrm{Na}^{+1}$ & $\mathrm{Ca}^{+2}$ & $\mathrm{Mg}^{+2}$ & $\mathrm{~K}^{+1}$ & $\mathrm{Cl}^{-1}$ & $\mathrm{SO}_{4}^{-2}$ & $\begin{aligned} & \mathrm{CO}_{3}^{-2} \\
&+ \mathrm{HCO}_{3}^{-1} \\
&\end{aligned}$ & & & & & \\
\hline \multirow{4}{*}{1} & $0-20$ & 7.8 & 119.1 & 136 & 27 & 8 & 4 & 184 & 13 & 0.5 & 15 & 14 & 45 & 6 & 126 \\
\hline & $20-50$ & 8.1 & 88.6 & 109 & 30 & 5 & 2 & 172 & 6 & 0.4 & 10 & 13 & 39 & 3 & 112 \\
\hline & $50-90$ & 7.7 & 103.4 & 185 & 26 & 7 & 4 & 223 & 16 & 0.4 & 12 & 15 & 43 & 3 & 78 \\
\hline & $90-150$ & 7.6 & 104.8 & 160 & 26 & 9 & 4 & 210 & 14 & 0.5 & 9 & 16 & 21 & 4 & 66 \\
\hline \multirow{3}{*}{2} & $0-30$ & 7.9 & 5.4 & 4 & 2 & 1 & 1 & 8 & 1 & 0.4 & 11 & 9 & 40 & 7 & 101 \\
\hline & $30-70$ & 9.4 & 3.1 & 4 & 1 & 0 & 0 & 6 & 1 & 0.2 & 12 & 8 & 40 & 9 & 69 \\
\hline & $70-130$ & 8.2 & 10.9 & 10 & 3 & 2 & 1 & 17 & 1 & 0.6 & 10 & 10 & 25 & 6 & 78 \\
\hline \multirow{4}{*}{3} & $0-15$ & 8.0 & 38.2 & 67 & 6 & 2 & 2 & 79 & 3 & 0.3 & 14 & 15 & 45 & 8 & 67 \\
\hline & \begin{tabular}{|l}
$15-55$ \\
\end{tabular} & 9.1 & 31.7 & 36 & 8 & 2 & 1 & 46 & 5 & 0.6 & 9 & 11 & 38 & 7 & 95 \\
\hline & $55-105$ & 8.6 & 57.3 & 82 & 14 & 3 & 1 & 102 & 8 & 0.4 & 11 & 13 & 43 & 5 & 68 \\
\hline & \begin{tabular}{|l}
$105-150$ \\
\end{tabular} & 8.4 & 44.2 & 57 & 10 & 3 & 2 & 81 & 3 & 0.8 & 8 & 14 & 23 & 5 & 55 \\
\hline \multirow{4}{*}{4} & $0-20$ & 8.2 & 41.8 & 49 & 14 & 4 & 2 & 80 & 2 & 0.4 & 16 & 12 & 50 & 6 & 44 \\
\hline & \begin{tabular}{|l|}
$20-70$ \\
\end{tabular} & 8.5 & 23.3 & 35 & 6 & 2 & 1 & 46 & 2 & 0.7 & 7 & 8 & 16 & 7 & 89 \\
\hline & \begin{tabular}{|l}
$70-110$ \\
\end{tabular} & 8.9 & \begin{tabular}{|l|}
55.4 \\
\end{tabular} & 56 & 17 & 8 & 2 & 101 & 3 & 0.6 & 7 & 14 & 17 & 4 & 56 \\
\hline & \begin{tabular}{|l}
$10-150$ \\
\end{tabular} & 8.8 & \begin{tabular}{|l|}
89.9 \\
\end{tabular} & 116 & 37 & 13 & 4 & 204 & 8 & 0.5 & 8 & 16 & 12 & 5 & 55 \\
\hline \multirow{3}{*}{5} & $0-30$ & 8.2 & 3.3 & 2 & 1 & 1 & 0 & 5 & 0 & 0.6 & 17 & 9 & 55 & 5 & 51 \\
\hline & $30-80$ & 8.0 & \begin{tabular}{|l|}
26.9 \\
\end{tabular} & 39 & 6 & 5 & 2 & 58 & 2 & 0.4 & 16 & 8 & 46 & 2 & 126 \\
\hline & \begin{tabular}{|l}
$80-140$ \\
\end{tabular} & 7.9 & 33.8 & 61 & 9 & 4 & 2 & 84 & 3 & 0.8 & 11 & 11 & 40 & 4 & 123 \\
\hline \multirow{4}{*}{6} & $0-25$ & 7.1 & \begin{tabular}{|l|}
24.9 \\
\end{tabular} & 23 & 7 & 4 & 2 & 41 & 2 & 0.3 & 11 & 6 & 40 & 13 & 78 \\
\hline & $25-60$ & 7.8 & 23.3 & 28 & 10 & 2 & 2 & 48 & 2 & 1.4 & 11 & 6 & 38 & 8 & 90 \\
\hline & \begin{tabular}{|c|}
$60-90$ \\
\end{tabular} & 7.9 & \begin{tabular}{|l|}
20.1 \\
\end{tabular} & 24 & 4 & 3 & 1 & 34 & 3 & 0.3 & 6 & 8 & 12 & 5 & 71 \\
\hline & \begin{tabular}{|l|}
$90-150$ \\
\end{tabular} & 7.8 & \begin{tabular}{|l|}
20.1 \\
12.9
\end{tabular} & 13 & 5 & 2 & 1 & 26 & 1 & 0.3 & 3 & 12 & 11 & 6 & 59 \\
\hline \multirow{3}{*}{7} & $0-30$ & 8.3 & 3.2 & 3 & 1 & 0 & 1 & 5 & 1 & 0.4 & 11 & 9 & 39 & 7 & 106 \\
\hline & $30-80$ & 8.5 & \begin{tabular}{|l|}
13.3 \\
\end{tabular} & 18 & 2 & 1 & 1 & 21 & 1 & 0.5 & 14 & 8 & 42 & 9 & 67 \\
\hline & \begin{tabular}{|l}
$80-140$ \\
\end{tabular} & 7.7 & \begin{tabular}{|l|}
27.4 \\
\end{tabular} & 34 & 11 & 2 & 1 & 56 & 2 & 0.5 & 10 & 7 & 37 & 6 & 93 \\
\hline \multirow{4}{*}{8} & $0-25$ & 8.4 & 17.2 & 26 & 7 & 2 & 2 & 43 & 1 & 0.8 & 10 & 5 & 40 & 7 & 56 \\
\hline & \begin{tabular}{|l|}
$25-70$ \\
\end{tabular} & 7.9 & 23.4 & 22 & 6 & 4 & 2 & 39 & 3 & 0.8 & 11 & 6 & 32 & 6 & 78 \\
\hline & \begin{tabular}{|l|}
$70-100$ \\
\end{tabular} & 8.7 & 41.5 & 45 & 12 & 3 & 2 & 70 & 3 & 0.6 & 9 & 12 & 34 & 8 & 82 \\
\hline & \begin{tabular}{|l|}
$100-150$ \\
\end{tabular} & 8.9 & \begin{tabular}{|l|}
39.8 \\
\end{tabular} & 48 & 11 & 3 & 2 & 77 & 3 & 0.6 & 8 & 11 & 27 & 4 & 112 \\
\hline & $0-30$ & 8.2 & \begin{tabular}{|l|}
10.7 \\
\end{tabular} & 13 & 3 & 1 & 1 & 18 & 1 & 0.4 & 13 & 9 & 39 & 6 & 72 \\
\hline 9 & $30-80$ & 8.5 & \begin{tabular}{|l|}
45.6 \\
\end{tabular} & 55 & 19 & 6 & 3 & 99 & 4 & 0.7 & 9 & 11 & 34 & 8 & 95 \\
\hline & \begin{tabular}{|l|}
$80-140$ \\
\end{tabular} & 8.4 & \begin{tabular}{|l|}
51.4 \\
\end{tabular} & 47 & 19 & 6 & 3 & 95 & 3 & 0.8 & 8 & 12 & 28 & 4 & 102 \\
\hline & $0-30$ & 8.3 & 33.5 & 31 & 9 & 5 & 2 & 59 & 2 & 0.6 & 9 & 8 & 26 & 8 & 67 \\
\hline 10 & $30-90$ & 8.7 & 66.2 & 75 & 24 & 10 & 4 & 146 & 2 & 0.5 & 13 & 16 & 42 & 4 & 79 \\
\hline & \begin{tabular}{|l}
$90-140$ \\
\end{tabular} & 8.6 & \begin{tabular}{|l|}
83.5 \\
\end{tabular} & 98 & 30 & 8 & 4 & 167 & 6 & 0.4 & 12 & 15 & 44 & 5 & 88 \\
\hline & $0-30$ & 8.2 & 4.1 & 3 & 1 & 1 & 0 & 6 & 1 & 0.2 & 12 & 14 & 39 & 9 & 49 \\
\hline 1 & $30-70$ & 8.1 & 11.4 & 13 & 3 & 1 & 1 & 20 & 1 & 0.6 & 15 & 9 & 41 & 3 & 115 \\
\hline 11 & \begin{tabular}{|l}
$70-120$ \\
\end{tabular} & 8.9 & \begin{tabular}{|l|}
18.1 \\
\end{tabular} & 21 & 6 & 2 & 1 & 34 & 1 & 0.8 & 11 & 5 & 38 & 10 & 133 \\
\hline & $20-150$ & 9.1 & 24.8 & 32 & 6 & 2 & 1 & 47 & 1 & 0.6 & 10 & 8 & 26 & 8 & 78 \\
\hline & $0-25$ & 8.2 & 22.7 & 29 & 6 & 4 & 2 & 45 & 2 & 0.5 & 18 & 6 & 57 & 10 & 149 \\
\hline 1) & \begin{tabular}{|l|}
$25-55$ \\
\end{tabular} & 8.0 & 18.9 & 23 & 4 & 3 & 2 & 34 & 2 & 0.4 & 11 & 5 & 34 & 10 & 124 \\
\hline 12 & \begin{tabular}{|l}
$55-110$ \\
\end{tabular} & 8.9 & 55.4 & 77 & 23 & 5 & 3 & 129 & 3 & 1.0 & 16 & 15 & 41 & 5 & 78 \\
\hline & \begin{tabular}{|l}
$110-150$ \\
\end{tabular} & 8.3 & 33.6 & 33 & 10 & 4 & 2 & 58 & 2 & 0.6 & 8 & 9 & 26 & 4 & 67 \\
\hline
\end{tabular}

The soil fertility of the study area is low where the available nitrogen content is low $(11-57 \mathrm{mg} / \mathrm{kg})$, the available phosphorus varies between 2 and 13 $\mathrm{mg} / \mathrm{kg}$ and the available potassium has low values of 44 and $149 \mathrm{mg} / \mathrm{kg}$. Therefore, these soils need NPK fertilizers to be added. 

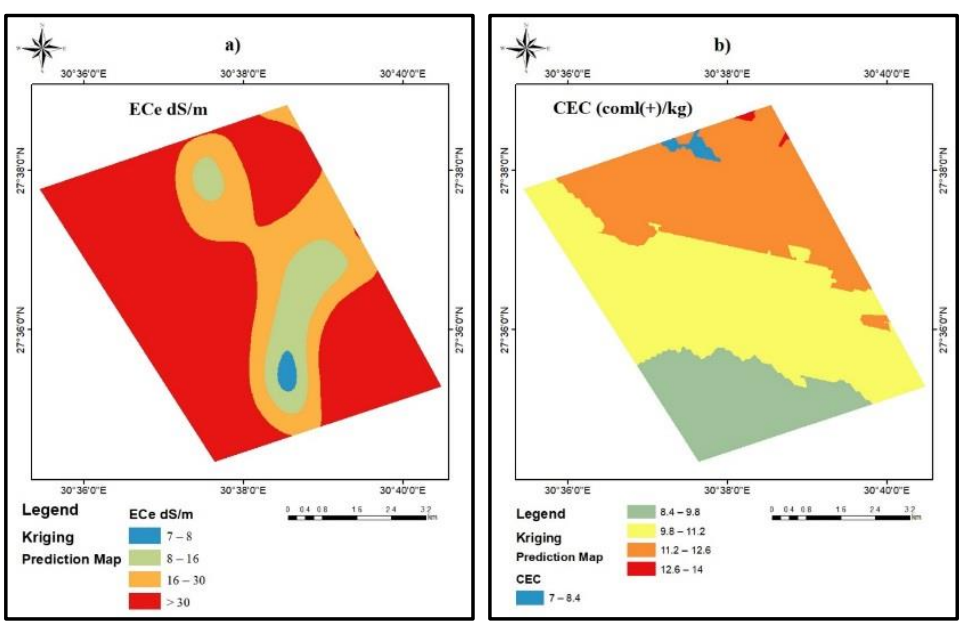

Figure (3): Spatial variability of a) the soil salinity $\left(\mathrm{EC}_{\mathrm{e}}\right)$ and $\mathrm{b}$ ) cation exchange capacity (CEC) of the study area.

\subsection{Land capability}

\subsubsection{ASLE program}

According to the applied system of land evaluation (ASLE) program, the obtained results showed that the study area has three classes of land capability, namely poor, very poor, and non-agricultural (Table 5 and Figure 4). Most of the investigated area are poor (C4). Soil profiles Nos. 1, 8, and 12 are very poor (C5), whereas soil profiles Nos. 3, 4, 9, and 10 are non-agricultural (C6).

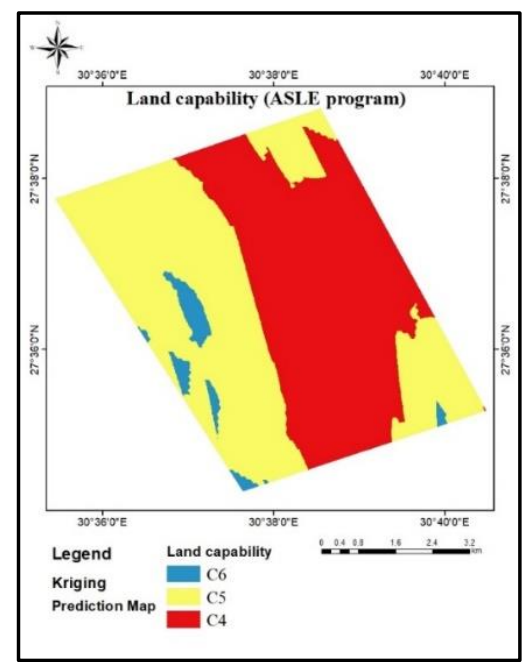

Figure (4): Land capability classes of the study area using the ASLE program. 
Table (5): Land capability classes of the investigated soils using the applied system of land evaluation (ASLE) program.

\begin{tabular}{|c|c|c|}
\hline \multirow{2}{*}{ Profile No. } & \multicolumn{2}{|c|}{ The applied system of land evaluation (ASLE) program } \\
\cline { 2 - 3 } & $\%$ & Class \\
\hline 1 & 10.09 & C5 (Very poor) \\
\hline 2 & 34.78 & C4 (Poor) \\
\hline 3 & 9.64 & C6 (Non-agricultural) \\
\hline 4 & 9.67 & C6 (Non-agricultural) \\
\hline 5 & 31.22 & C4 (Poor) \\
\hline 6 & 25.78 & C4 (Poor) \\
\hline 7 & 32.65 & C4 (Poor) \\
\hline 8 & 11.28 & C5 (Very poor) \\
\hline 9 & 9.50 & C6 (Non-agricultural) \\
\hline 10 & 6.83 & C6 (Non-agricultural) \\
\hline 11 & 34.67 & C4 (Poor) \\
\hline 12 & 12.69 & C5 (Very poor) \\
\hline
\end{tabular}

\subsubsection{MicroLEIS (Cervatana model)}

soil salinity, soil fertility, and erosion risk.

Concerning the application of the microcomputer land evaluation information system (MicroLEISCervatana model), all the study areas have a marginal capability grade, except soil profile No. 2 which has a moderate capability grade (Table 6 and Figure 5). The soil limitations are coarse soil texture,

\subsection{Land suitability}

\subsubsection{ASLE program}

The land suitability classes for some selected crops using the applied system of land evaluation program that can be grown in the study area are shown in Table (7) and Figure (6).

Table (6): Land capability grades of the studied soils using MicroLEIS program (Cervatana model).

\begin{tabular}{|c|c|}
\hline \multirow{2}{*}{ Profile No. } & MicroLEIS program (Cervatana model) \\
\cline { 2 - 2 } & Grade \\
\hline 1 & N1(Marginal) \\
\hline 2 & S3r (Moderate) \\
\hline 3 & N1(Marginal) \\
\hline 4 & N1(Marginal) \\
\hline 5 & N1(Marginal) \\
\hline 6 & N1(Marginal) \\
\hline 7 & N1(Marginal) \\
\hline 8 & N1(Marginal) \\
\hline 9 & N1(Marginal) \\
\hline 10 & N1(Marginal) \\
\hline 11 & N1(Marginal) \\
\hline 12 & N1(Marginal) \\
\hline
\end{tabular}

\footnotetext{
r: Erosion risk, 1: Soil limitation.
} 


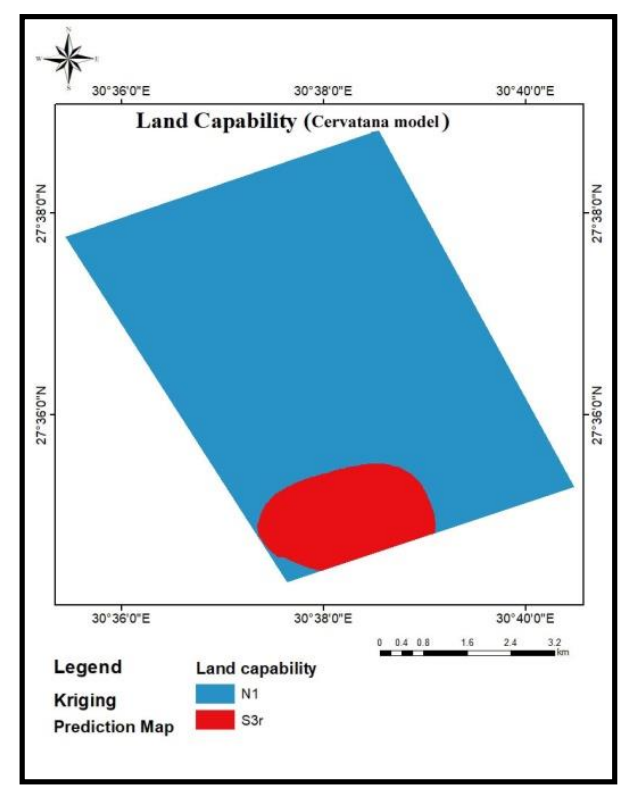

Figure (5): Land capability classes of the study area using MicroLEIS program (Cervatana model).

Accordingly, the soils of the study area have in general a widely range of suitability grades, from highly suitable (S1) to not suitable $\mathrm{N}$ (currently not suitable, N1 and permanently not suitable, N2). Most of the selected crops are highly suitable, suitable moderately suitable, and marginally suitable for field crops, forage crops, vegetables, and fruit crops, except crops rice, cabbage, and banana that are currently not suitable (N1) and permanently not suitable (N2) for some soils.

Table (7): Land suitability classes under the surface, sprinkler, and drip irrigation systems of the study area for selected crops using ASLE program.

\begin{tabular}{|c|c|c|c|c|c|c|c|c|c|c|c|c|c|c|c|c|c|c|c|c|c|c|c|c|c|c|c|c|}
\hline \multirow[b]{2}{*}{ 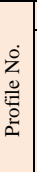 } & \multicolumn{11}{|c|}{ Field Crops } & \multicolumn{2}{|c|}{ Forage Crops } & \multicolumn{7}{|c|}{ Vegetables } & \multicolumn{8}{|c|}{ Fruit Crops } \\
\hline & 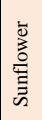 & 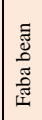 & 厄. & 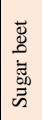 & $\begin{array}{l}\vec{\varpi} \\
\frac{\tilde{J}}{3}\end{array}$ & 这 & 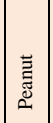 & $\frac{\mathbb{N}}{\tilde{N}}$ & 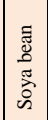 & 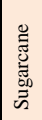 & $\stackrel{\mathscr{v}}{\sim}$ & 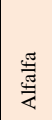 & $\begin{array}{l}\text { E } \\
\text { 咅 } \\
\bar{b} \\
\dot{b}\end{array}$ & 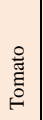 & 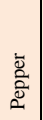 & ळ & 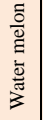 & 离 & 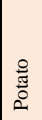 & 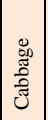 & 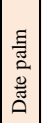 & 架 & $\stackrel{0}{\stackrel{0}{0}}$ & 总 & 总 & 离 & 总 & $\begin{array}{l}\text { 駕 } \\
\text { 荧 }\end{array}$ \\
\hline 1 & $\mathrm{~S}_{4}$ & $\mathrm{NS}_{1}$ & $\mathrm{NS}_{2}$ & $\mathrm{NS}_{2}$ & $\mathrm{NS}_{2}$ & $\mathrm{NS}_{1}$ & $\mathrm{NS}_{1}$ & $\mathrm{NS}_{1}$ & $\mathrm{NS}_{1}$ & $\mathrm{NS}_{2}$ & $\mathrm{NS}_{2}$ & $\mathrm{NS}_{2}$ & $\mathrm{NS}_{1}$ & $\mathrm{~S}_{4}$ & $\mathrm{~S}_{4}$ & $\mathrm{~S}_{4}$ & $\mathrm{~S}_{4}$ & $\mathrm{NS}_{2}$ & $\mathrm{NS}_{1}$ & $\mathrm{NS}_{2}$ & $\mathrm{~S}_{4}$ & $\mathrm{~S}_{4}$ & $\mathrm{~S}_{4}$ & $\mathrm{~S}_{4}$ & $\mathrm{NS}_{1}$ & $\mathrm{NS}_{2}$ & $\mathrm{NS}_{1}$ & IS \\
\hline 2 & $S_{2}$ & $\mathrm{~S}_{3}$ & $\mathrm{~S}_{4}$ & $\mathrm{~S}_{4}$ & $\mathrm{~S}_{4}$ & $\mathrm{~S}_{2}$ & $\mathrm{~S}_{2}$ & $\mathrm{~S}_{3}$ & $\mathrm{~S}_{3}$ & $\mathrm{~S}_{4}$ & $\mathrm{NS}_{2}$ & $\mathrm{~S}_{4}$ & $\mathrm{~S}_{3}$ & $\mathrm{~S}_{2}$ & $\mathrm{~S}_{2}$ & $\mathrm{~S}_{2}$ & $\mathrm{~S}_{2}$ & $\mathrm{~S}_{4}$ & $\mathrm{~S}_{3}$ & $\mathrm{NS}_{1}$ & $\mathrm{~S}_{2}$ & $\mathrm{~S}_{2}$ & $\mathrm{~S}_{2}$ & $\begin{array}{ll}S_{2} \\
\end{array}$ & $\mathrm{~S}_{3}$ & $\mathrm{~S}_{4}$ & $\mathrm{~S}_{2}$ & t. \\
\hline 3 & $\mathrm{~S}_{4}$ & $\mathrm{NS}_{1}$ & $\mathrm{NS}_{2}$ & $\mathrm{NS}_{2}$ & $\mathrm{NS}_{2}$ & $\mathrm{NS}_{1}$ & $\mathrm{NS}_{1}$ & $\mathrm{NS}_{1}$ & $\mathrm{NS}_{1}$ & $\mathrm{NS}_{2}$ & $\mathrm{Ns}$ & $\mathrm{NS}_{2}$ & $\mathrm{NS}_{1}$ & $\mathrm{~S}_{4}$ & $\mathrm{~S}_{4}$ & $\mathrm{~S}_{4}$ & $\mathrm{~S}_{4}$ & $\mathrm{NS}_{2}$ & $\mathrm{NS}_{1}$ & $\mathrm{NS}_{2}$ & $\mathrm{~S}_{4}$ & $\mathrm{~S}_{4}$ & $S_{4}$ & \begin{tabular}{l|l}
$S_{4}$ \\
\end{tabular} & $\mathrm{NS}_{1}$ & $\mathrm{~S}_{2}$ & & \\
\hline 4 & $\mathrm{NS}_{1}$ & $\mathrm{NS}_{1}$ & $\mathrm{NS}_{2}$ & $\mathrm{NS}_{2}$ & $\mathrm{NS}_{2}$ & $\mathrm{NS}_{1}$ & $\mathrm{NS}_{1}$ & $\mathrm{NS}_{1}$ & $\mathrm{NS}_{1}$ & $\mathrm{NS}_{2}$ & $\mathrm{NS}_{2}$ & $\mathrm{NS}_{2}$ & $\mathrm{NS}_{1}$ & $\mathrm{~S}_{4}$ & $\mathrm{~S}_{4}$ & $\mathrm{NS}_{1} \mid \mathrm{I}$ & $\mathrm{NS}_{1}$ & $\mathrm{NS}_{2}$ & $\mathrm{NS}_{1}$ & $\mathrm{NS}_{2}$ & $\mathrm{~S}_{4}$ & $\mathrm{NS}_{1}$ & $\mathrm{NS}_{1}$ & $\mathrm{NS}_{1}$ & $\mathrm{NS}_{1}$ & $\mathrm{NS}_{2}$ & $\mathrm{NS}_{1}$ & \\
\hline 5 & $\mathrm{~S}_{2}$ & $\mathrm{NS}_{1}$ & $\mathrm{~S}_{4}$ & $\mathrm{~S}_{4}$ & $\mathrm{~S}_{4}$ & \begin{tabular}{|l|}
$\mathrm{S}_{3}$ \\
\end{tabular} & $\mathrm{NS}_{1}$ & $\mathrm{NS}_{1}$ & $\mathrm{NS}_{1}$ & $\mathrm{~S}_{4}$ & $\mathrm{NS}_{2}$ & $\mathrm{~S}_{4}$ & $\mathrm{~S}_{3}$ & $\mathrm{~S}_{2}$ & $\mathrm{~S}_{4}$ & $\mathrm{NS}_{1} \mathrm{I}$ & $\mathrm{NS}_{1}$ & $\mathrm{NS}_{2}$ & $\mathrm{NS}_{1}$ & $\mathrm{NS}_{2}$ & $\mathrm{~S}_{2}$ & $\mathrm{~S}_{2}$ & \begin{tabular}{|l|}
$\mathrm{S}_{2}$ \\
\end{tabular} & \begin{tabular}{|l|}
$\mathrm{S}_{2}$ \\
\end{tabular} & $\mathrm{NS}_{1}$ & \begin{tabular}{|l|l|}
$\mathrm{NS}_{2}$ & \\
\end{tabular} & $\mathrm{NS}_{1}$ & 1 \\
\hline 6 & $\begin{array}{ll}S_{4} \\
\end{array}$ & $\mathrm{NS}_{2}$ & $\mathrm{~S}_{4}$ & $\mathrm{~S}_{4}$ & $\mathrm{NS}_{2}$ & $\mathrm{~S}_{4}$ & $\mathrm{NS}_{1}$ & $\mathrm{NS}_{2}$ & $\mathrm{NS}_{2}$ & $\mathrm{NS}_{2}$ & $\mathrm{NS}_{2}$ & $\mathrm{NS}_{2}$ & $\mathrm{NS}_{2}$ & $\mathrm{NS}_{2}$ & $\mathrm{NS}_{2}$ & \begin{tabular}{|l|l}
$\mathrm{NS}_{2}$ & $\mathrm{I}$ \\
\end{tabular} & $\mathrm{NS}_{2}$ & $\mathrm{NS}_{2}$ & $\mathrm{NS}_{1}$ & $\mathrm{NS}_{2}$ & $\mathrm{~S}_{2}$ & $\mathrm{~S}_{4}$ & $\mathrm{NS}_{2}$ & $\begin{array}{ll}S_{4} \\
\end{array}$ & $\begin{array}{lll}\mathrm{NS}_{2} & \\
\end{array}$ & \begin{tabular}{|l|l}
$\mathrm{NS}_{2}$ & \\
\end{tabular} & $\mathrm{NS}_{1}$ & NS \\
\hline 7 & $S_{2}$ & $\mathrm{NS}_{1}$ & $\begin{array}{l}\mathrm{S}_{4} \\
\end{array}$ & $\mathrm{~S}_{4}$ & $\mathrm{~S}_{4}$ & $\mathrm{~S}_{2}$ & $\mathrm{~S}_{3}$ & $\mathrm{~S}_{3}$ & $\mathrm{NS}_{1}$ & $\mathrm{~S}_{4}$ & $\mathrm{NS}_{2}$ & $\mathrm{~S}_{4}$ & $\mathrm{~S}_{3}$ & $\mathrm{~S}_{2}$ & $\mathrm{~S}_{2}$ & \begin{tabular}{|l|l}
$\mathrm{NS}_{1}$ \\
\end{tabular} & $\mathrm{~S}_{3}$ & $\mathrm{~S}_{4}$ & $\mathrm{~S}_{3}$ & $\mathrm{NS}_{2}$ & $\mathrm{~S}_{2}$ & $\mathrm{~S}_{2}$ & \begin{tabular}{|l|}
$\mathrm{S}_{2}$ \\
\end{tabular} & $\mathrm{~S}_{2}$ & $\mathrm{~S}_{3}$ & $\mathrm{NS}_{1}$ & \begin{tabular}{|l|}
$S_{3}$ \\
\end{tabular} & NS \\
\hline 8 & $S_{4}$ & $\mathrm{~S}_{4}$ & $\mathrm{~S}_{4}$ & $\mathrm{NS}_{2}$ & $\mathrm{NS}_{2}$ & $\mathrm{~S}_{2}$ & $\mathrm{NS}_{1}$ & $\mathrm{NS}_{1}$ & $\mathrm{NS}_{1}$ & $\mathrm{NS}_{2}$ & $\mathrm{NS}_{2}$ & $\mathrm{NS}_{2}$ & $\mathrm{NS}_{1}$ & $\mathrm{~S}_{4}$ & $\mathrm{~S}_{4}$ & $\mathrm{~S}_{4}$ & $\mathrm{~S}_{4}$ & $\mathrm{NS}_{2}$ & $\mathrm{NS}_{1}$ & $\mathrm{NS}_{2}$ & $\mathrm{~S}_{2}$ & $\mathrm{~S}_{4}$ & $\mathrm{~S}_{4}$ & \begin{tabular}{l|}
$S_{4}$ \\
\end{tabular} & $\mathrm{NS}_{1}$ & $\mathrm{NS}_{2}$ & $\mathrm{NS}_{1}$ & $\mathrm{NS}$ \\
\hline 9 & $\begin{array}{l}S_{3} \\
\end{array}$ & $\mathrm{NS}_{1}$ & $S_{4}$ & $\begin{array}{l}S_{4} \\
\end{array}$ & $\mathrm{NS}_{2}$ & $\mathrm{~S}_{3}$ & $\mathrm{NS}_{1}$ & $\mathrm{NS}_{1}$ & $\mathrm{NS}_{1}$ & $\mathrm{NS}_{2}$ & $\mathrm{NS}_{2}$ & $\mathrm{NS}_{2}$ & $\mathrm{NS}_{1}$ & $\mathrm{~S}_{4}$ & $\begin{array}{ll}S_{4} \\
\end{array}$ & $\begin{array}{lll}N_{1} & \\
\end{array}$ & $\mathrm{NS}_{1}$ & $\mathrm{NS}_{2}$ & $\mathrm{NS}_{1}$ & $\mathrm{NS}_{2}$ & $\mathrm{~S}_{2}$ & $S_{4}$ & $\mathrm{NS}_{1}$ & \begin{tabular}{|l|}
$\mathrm{S}_{4}$ \\
\end{tabular} & $\mathrm{NS}_{1}$ & $S_{2}$ & $\mathrm{SS}_{1}$ & \\
\hline 10 & $\mathrm{~S}$ & $\mathrm{NS}_{2}$ & $\mathrm{~S}_{4}$ & $\mathrm{~S}_{4}$ & $\mathrm{~S}_{4}$ & $\mathrm{~S}_{4}$ & $\mathrm{NS}_{1}$ & $\mathrm{NS}_{2}$ & $\mathrm{NS}_{2}$ & $\mathrm{NS}_{1}$ & $\mathrm{NS}_{2}$ & $\mathrm{NS}_{2}$ & $\mathrm{NS}_{2}$ & $\mathrm{NS}_{2}$ & $\mathrm{NS}_{2}$ & $\mathrm{NS}_{2}$ & $\mathrm{NS}_{2}$ & $\mathrm{NS}_{2}$ & $\mathrm{NS}_{1}$ & $\mathrm{NS}_{2}$ & $\mathrm{~S}_{2}$ & $\mathrm{~S}_{3}$ & \begin{tabular}{|l|}
$\mathrm{S}_{4}$ \\
\end{tabular} & $\mathrm{NS}_{1}$ & $\mathrm{NS}_{2}$ & $\mathrm{NS}_{2}$ & $\mathrm{NS}_{1}$ & $\mathrm{~S}_{2}$ \\
\hline 11 & $S_{1}$ & $\mathrm{~S}_{1}$ & $S_{1}$ & $\mathrm{~S}_{1}$ & $S_{1}$ & $\mathrm{~S}_{2}$ & $\mathrm{~S}_{2}$ & $\mathrm{~S}_{2}$ & \begin{tabular}{|l|}
$\mathrm{S}_{2}$ \\
\end{tabular} & $\mathrm{~S}_{2}$ & $\mathrm{NS}_{2}$ & $\mathrm{~S}_{1}$ & $\mathrm{~S}_{2}$ & $\mathrm{~S}_{1}$ & $\mathrm{~S}_{1}$ & $S_{1}$ & $S_{1}$ & $\begin{array}{ll}S_{1} \\
\end{array}$ & $\mathrm{~S}_{2}$ & $\mathrm{NS}_{2}$ & $S_{1}$ & $S_{1}$ & $\mathrm{~S}_{1}$ & \begin{tabular}{|l|}
$S_{1}$ \\
\end{tabular} & \begin{tabular}{|l|}
$S_{2}$ \\
\end{tabular} & \begin{tabular}{|l|}
$S_{2}$ \\
\end{tabular} & $S_{1}$ & $\sqrt{S}$ \\
\hline 12 & \begin{tabular}{|l|}
$\mathrm{S}_{2}$ \\
\end{tabular} & $\mathrm{~S}_{2}$ & \begin{tabular}{ll|}
$\mathrm{S}_{4}$ \\
\end{tabular} & $\mathrm{~S}_{4}$ & $\mathrm{~S}_{4}$ & $\mathrm{~S}_{2}$ & $\begin{array}{l}\mathrm{S}_{2} \\
\end{array}$ & $S_{2}$ & \begin{tabular}{|l|}
$\mathrm{S}_{2}$ \\
\end{tabular} & $\mathrm{~S}_{4}$ & $\mathrm{NS}_{2}$ & $\mathrm{~S}_{4}$ & $S_{2}$ & $\mathrm{~S}_{2}$ & $\mathrm{~S}_{2}$ & $\mathrm{~S}_{2}$ & $\begin{array}{l}S_{2} \\
\end{array}$ & \begin{tabular}{|l|}
$\mathrm{S}_{3}$ \\
\end{tabular} & $\mathrm{~S}_{2}$ & $\mathrm{NS}_{1}$ & \begin{tabular}{|l|}
$S_{1}$ \\
\end{tabular} & $\mathrm{~S}_{1}$ & $\begin{array}{ll}S_{1} \\
\end{array}$ & \begin{tabular}{|l|}
$\mathrm{S}_{2}$ \\
\end{tabular} & \begin{tabular}{|l|}
$\mathrm{S}_{2}$ \\
\end{tabular} & \begin{tabular}{l|l}
$\mathrm{S}_{4}$ \\
\end{tabular} & \begin{tabular}{|l|}
$\mathrm{S}_{2}$ \\
\end{tabular} & $\mathrm{NS}$ \\
\hline
\end{tabular}

S1: Highly suitable (>80\%), S2: Suitable (60-80\%), S3: Moderately suitable (30-60\%), S4: Marginally suitable 
(20-30\%), N1: Currently not suitable (10-20\%), N2: Permanently not suitable $(<10 \%)$.
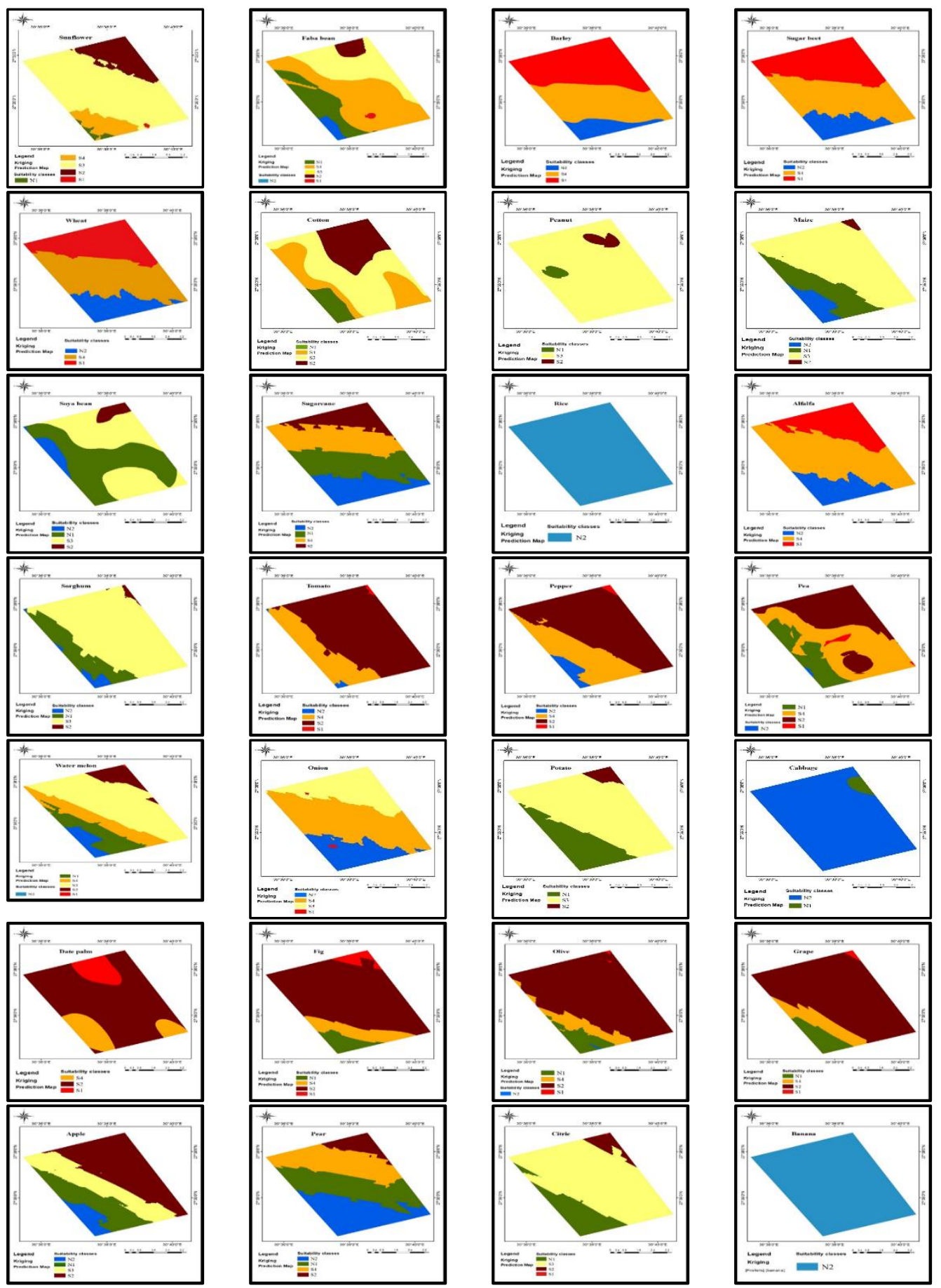

Figure (6): Land suitability of the study area for selected crops using ASLE program. 


\subsubsection{MicroLEIS (Almagra model)}

In general, the soils of the study area are not suitable (5) for the selected crops using the microcomputer land evaluation information system (MicroLEIS-Almagra model) with few exceptional cases which are present and illustrated in Table (8) and Figure (7) respectively. The soil limitations of the current study are soil texture, soil salinity, and soil fertility.
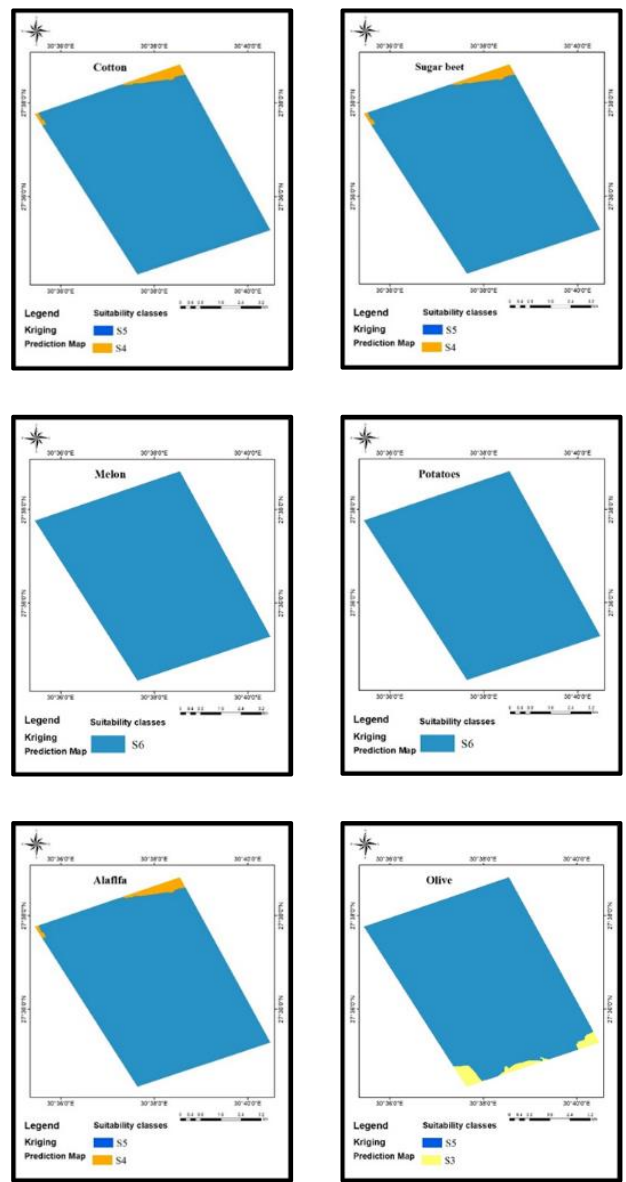

\subsection{Geostatistical analysis}

Geostatistical analyses for mapping distribution of some soil properties, land capability, and land suitability rates were calculated using variance structure that was performed using eleven semivariogram models; circular, spherical, tetraspherical, pentaspherical, exponential, gaussian, rational quadratic, hole effect, kbessel, j-bessel and stable.
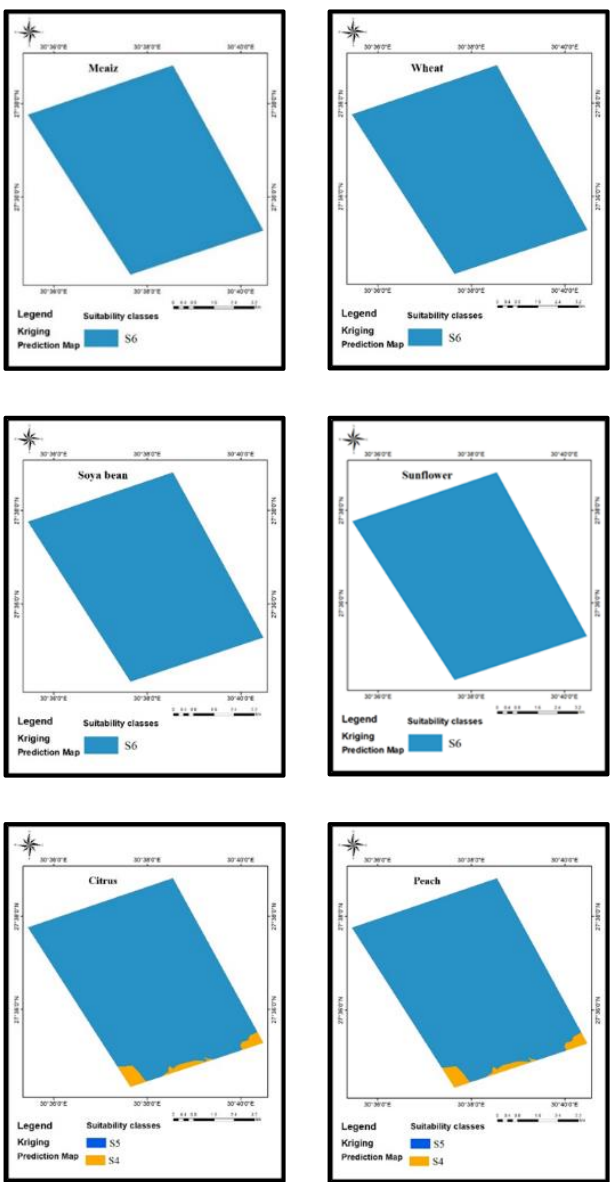

Figure (7): Land suitability of the study area for some selected crops using MicroLIES (Almagra model). 
Table (8): Land suitability classes of the study area for some selected crops using the MicroLIES (Almagra model) program.

\begin{tabular}{|c|c|c|c|c|c|c|c|c|c|c|c|c|}
\hline \multirow{2}{*}{ Profile No. } & \multicolumn{8}{|c|}{ Annual crops } & \multirow{2}{*}{\begin{tabular}{|c|} 
Semi-annual crops \\
Alfalfa
\end{tabular}} & \multicolumn{3}{|c|}{ Perennial crops } \\
\hline & Cotton & Sugar-beet & Maize & Wheat & Melon & Potatoes & Soya- bean & Sunflower & & Olive & Citrus & Peach \\
\hline 1 & $\mathrm{~S}_{5}$ ts & S $_{5}$ ts & $\mathrm{S}_{5}$ ts & $\mathrm{S}_{5}$ ts & S sts & $\mathrm{S}_{5}$ ts & S S $_{5}$ ts & $\mathrm{S}_{5} \mathrm{ts}$ & $\mathrm{S}_{5}$ ts & S Sts & $\mathrm{S}_{5}$ ts & $\mathrm{S}_{5}$ ts \\
\hline 2 & $\mathrm{~S}_{5} \mathrm{t}$ & $\mathrm{S}_{5} \mathrm{t}$ & $S_{5} t$ & $\mathrm{~S}_{5} \mathrm{t}$ & $\mathrm{S}_{5} \mathrm{t}$ & $\mathrm{S}_{5} \mathrm{t}$ & $\mathrm{S}_{5} \mathrm{t}$ & $\mathrm{S}_{5} \mathrm{t}$ & $\mathrm{S}_{5} \mathrm{t}$ & $\mathrm{S}_{3} \mathrm{ts}$ & $\mathrm{S}_{4} \mathrm{ts}_{\mathrm{s}}$ & $\mathrm{S}_{4} \mathrm{ts}_{\mathrm{s}}$ \\
\hline 3 & $\mathrm{~S}_{5} \mathrm{~S}$ & $\mathrm{~S}_{5} \mathrm{~S}$ & $\mathrm{~S}_{5} \mathrm{~S}$ & $\mathrm{~S}_{5} \mathrm{~S}$ & $\mathrm{~S}_{5} \mathrm{~S}$ & $\mathrm{~S}_{5} \mathrm{~S}$ & $\mathrm{~S}_{5} \mathrm{~S}$ & $\mathrm{~S}_{5} \mathrm{~S}$ & $\mathrm{~S}_{5} \mathrm{~S}$ & $\mathrm{~S}_{5} \mathrm{~s}$ & $\mathrm{~S}_{5} \mathrm{~s}$ & $\mathrm{~S}_{5} \mathrm{~s}$ \\
\hline 4 & $\mathrm{~S}_{5 \mathrm{~S}}$ & $\mathrm{~S}_{5} \mathrm{~S}$ & $\mathrm{~S}_{5} \mathrm{~s}$ & $\mathrm{~S}_{5 \mathrm{~S}}$ & $\mathrm{~S}_{5 \mathrm{~S}}$ & $\mathrm{~S}_{5} \mathrm{~s}$ & $\mathrm{~S}_{5 \mathrm{~S}}$ & $\mathrm{~S}_{5 \mathrm{~S}}$ & $\mathrm{~S}_{5 \mathrm{~S}}$ & $\mathrm{~S}_{5} \mathrm{~s}$ & $\mathrm{~S}_{5} \mathrm{~s}$ & $\mathrm{~S}_{5} \mathrm{~s}$ \\
\hline 5 & $\mathrm{~S}_{5} \mathrm{~S}$ & $\mathrm{~S}_{5} \mathrm{~S}$ & $\mathrm{~S}_{5} \mathrm{~S}$ & $\mathrm{~S}_{5} \mathrm{~S}$ & $\mathrm{~S}_{5} \mathrm{~S}$ & $\mathrm{~S}_{5} \mathrm{~s}$ & $\mathrm{~S}_{5} \mathrm{~S}$ & $\mathrm{~S}_{5} \mathrm{~S}$ & $\mathrm{~S}_{5} \mathrm{~S}$ & $\mathrm{~S}_{5} \mathrm{~S}$ & $\mathrm{~S}_{5} \mathrm{~S}$ & $\mathrm{~S}_{5} \mathrm{~S}$ \\
\hline 6 & $\mathrm{~S}_{5} \mathrm{~S}$ & $\mathrm{~S}_{5} \mathrm{~S}$ & $\mathrm{~S}_{5} \mathrm{~S}$ & $\mathrm{~S}_{5} \mathrm{~s}$ & $\mathrm{~S}_{5} \mathrm{~S}$ & $\mathrm{~S}_{5} \mathrm{~s}$ & $\mathrm{~S}_{5} \mathrm{~S}$ & $\mathrm{~S}_{5} \mathrm{~s}$ & $\mathrm{~S}_{5} \mathrm{~s}$ & $\mathrm{~S}_{5} \mathrm{~s}$ & $\mathrm{~S}_{5} \mathrm{~s}$ & $\mathrm{~S}_{5} \mathrm{~s}$ \\
\hline 7 & $\mathrm{~S}_{5} \mathrm{~S}$ & $\mathrm{~S}_{5 \mathrm{~S}}$ & $\mathrm{~S}_{5} \mathrm{~s}$ & $\mathrm{~S}_{5} \mathrm{~s}$ & $\mathrm{~S}_{5 \mathrm{~S}}$ & $\mathrm{~S}_{5} \mathrm{~s}$ & $\mathrm{~S}_{5 \mathrm{~S}}$ & $\mathrm{~S}_{5} \mathrm{~s}$ & $\mathrm{~S}_{5} \mathrm{~s}$ & $\mathrm{~S}_{5} \mathrm{~s}$ & $\mathrm{~S}_{5} \mathrm{~s}$ & $\mathrm{~S}_{5 \mathrm{~S}}$ \\
\hline 8 & $\mathrm{~S}_{5} \mathrm{~S}$ & $\mathrm{~S}_{5} \mathrm{~S}$ & $\mathrm{~S}_{5 \mathrm{~S}}$ & $\mathrm{~S}_{5} \mathrm{~S}$ & $\mathrm{~S}_{55}$ & $\mathrm{~S}_{5} \mathrm{~S}$ & $\mathrm{~S}_{5} \mathrm{~S}$ & $\mathrm{~S}_{5} \mathrm{~S}$ & $\mathrm{~S}_{5} \mathrm{~S}$ & $\mathrm{~S}_{5} \mathrm{~S}$ & $\mathrm{~S}_{5} \mathrm{~S}$ & $\mathrm{~S}_{5} \mathrm{~S}$ \\
\hline 9 & $\mathrm{~S}_{5} \mathrm{~S}$ & $\mathrm{~S}_{5} \mathrm{~S}$ & $\mathrm{~S}_{5} \mathrm{~s}$ & $\mathrm{~S}_{5} \mathrm{~S}$ & $\mathrm{~S}_{5} \mathrm{~S}$ & $\mathrm{~S}_{5} \mathrm{~S}$ & $\mathrm{~S}_{5} \mathrm{~S}$ & $\mathrm{~S}_{5} \mathrm{~S}$ & $\mathrm{~S}_{5} \mathrm{~s}$ & $\mathrm{~S}_{5} \mathrm{~S}$ & $\mathrm{~S}_{5} \mathrm{~S}$ & $\mathrm{~S}_{5} \mathrm{~S}$ \\
\hline 10 & $\mathrm{~S}_{5} \mathrm{~S}$ & $\mathrm{~S}_{5 \mathrm{~S}}$ & $\mathrm{~S}_{5 \mathrm{~S}}$ & $\mathrm{~S}_{55} \mathrm{~S}$ & $\mathrm{~S}_{5} \mathrm{~s}$ & $\mathrm{~S}_{5} \mathrm{~s}$ & $\mathrm{~S}_{55}$ & $\mathrm{~S}_{5} \mathrm{~s}$ & $\mathrm{~S}_{5} \mathrm{~S}$ & $\mathrm{~S}_{5} \mathrm{~S}$ & $\mathrm{~S}_{5} \mathrm{~S}$ & $\mathrm{~S}_{5 \mathrm{~S}}$ \\
\hline 11 & $\mathrm{~S}_{4} \mathrm{ts}$ & S4ts & $\mathrm{S}_{5 \mathrm{~S}}$ & $\mathrm{~S}_{55}$ & $\mathrm{~S}_{5 \mathrm{~S}}$ & $\mathrm{~S}_{55} \mathrm{~s}$ & $\mathrm{~S}_{5 \mathrm{~S}}$ & $\mathrm{~S}_{55} \mathrm{~s}$ & S4ts & $\mathrm{S}_{5} \mathrm{~S}$ & $\mathrm{~S}_{5 \mathrm{~S}}$ & $\mathrm{~S}_{55}$ \\
\hline 12 & $\mathrm{~S}_{5} \mathrm{~s}$ & $\mathrm{~S}_{5} \mathrm{~S}$ & $\mathrm{~S}_{5} \mathrm{~s}$ & $\mathrm{~S}_{5} \mathrm{~S}$ & $\mathrm{~S}_{5} \mathrm{~S}$ & $\mathrm{~S}_{5} \mathrm{~S}$ & $\mathrm{~S}_{5} \mathrm{~S}$ & $\mathrm{~S}_{5} \mathrm{~S}$ & $\mathrm{~S}_{5} \mathrm{~S}$ & $\mathrm{~S}_{5} \mathrm{~S}$ & $\mathrm{~S}_{5} \mathrm{~S}$ & $\mathrm{~S}_{5} \mathrm{~S}$ \\
\hline
\end{tabular}

S3: Moderately suitable, S4: Marginally suitable, S5: Non-suitable, t: soil texture, s: salinity.

Table (9): Geostatistical analyses of some soil parameters of the study area.

\begin{tabular}{|c|l|c|c|c|c|}
\hline \multirow{4}{*}{ Method } & Parameter & Model & Mean & $\begin{array}{c}\text { Mean } \\
\text { Standardized }\end{array}$ & $\begin{array}{c}\text { Root Mean Square } \\
\text { Standardized }\end{array}$ \\
\hline \multirow{5}{*}{$\begin{array}{c}\text { Ordinary } \\
\text { Kriging }\end{array}$} & Calcium carbonate & K-Bessel & -0.0633 & -0.0083 & 1.1270 \\
\cline { 2 - 5 } & Gypsum content & K-Bessel & -0.0216 & -0.0192 & 2.0195 \\
\cline { 2 - 5 } & Soil salinity & J-Bessel & 0.9990 & -0.1805 & 1.2543 \\
\cline { 2 - 5 } & Cation exchange capacity & Circular & -0.1575 & -0.0707 & 1.1288 \\
\cline { 2 - 6 } & Land capability by ASLE & J-Bessel & 1.9439 & 0.1601 & 1.2242 \\
\cline { 2 - 5 } & Land capability by MicroLEIS & J-Bessel & 0.8857 & 0.1823 & 1.9774 \\
\cline { 2 - 5 } & Land suitability using ASLE & Stable & -0.3242 & -0.0144 & 1.2039 \\
\cline { 2 - 5 } & Land suitability using MicroLEIS & Stable & -0.0763 & -0.0123 & 1.1958 \\
\hline
\end{tabular}

However, the best four models for evaluation were circular, J-Bessel, KBessel, and stable depending on values of mean prediction errors, root means square prediction errors, and mean standardized prediction errors which should be close to 0 and root mean square standardized prediction errors which must close to 1 (Table 9).

\section{Conclusion}

In conclusion, the assessment results of land capability and land suitability for the selected crops helps in planning sustainable agriculture programs. Integration between geographic information systems (GIS), ASLE, and MicroLIES programs was undertaken in these soils to assess the land performance. The results of these soils indicated that the major soil limitations are soil texture, soil salinity, and low soil fertility characteristics, which can be improved using good management practices such as adding organic matter, fertilizers for upgrade the fertility, leaching the excess salt, and good agriculture practices for crops. These improvements will develop the potential suitability. Ultimately, from this study, it can be mentioned that the geostatistical approach and GIS are effective and strong tools for land capability and suitability studies and hence for sustainable planning of land use.

\section{References}

AbdelRahman, M. A., Natarajan, A. and Hegde, R. (2016), "Assessment of land suitability and capability by integrating remote sensing and GIS for agriculture in Chamarajanagar district, Karnataka, India", The 
Egyptian Journal of Remote Sensing and Space Science, Vol. 19 No. 1, pp. 125-141.

Bandyopadhyay, S., Jaiswal, R. K., Hegde, V. S. and Jayaraman, V. (2009), "Assessment of land suitability potentials for agriculture using a remote sensing and GIS based approach", International Journal of Remote Sensing, Vol. 30 No. 4, pp. 879-895.

De la Rosa, D., Mayol, F., Diaz-Pereira, E. and Fernandez, M. (2009), "A land evaluation decision support system (MicroLEIS DSS) for agricultural soil protection", Environmental Modeling and Software, Vol. 19, pp. 929-942.

De la Rosa, D., Moreno, J. A., Garcia, L. V. and Almorza, J. (2004), "MicroLEIS: A microcomputerbased Mediterranean land evaluation information system", Soil Use and Management, Vol. 8, pp. 89-96.

ESRI (2014), Arc Map version 10.2.2. User Manual, 380 New York Street, Redlands, California, 92373-8100, USA.

Fadel, M. E. and Sayed, Y. A. (2020), "Land Resources Evaluation for Sustainable Agriculture in El-Qusiya Area, Assiut, Egypt. Egypt", Egyptian Journal of Soil Science, Vol. 60 No. 3, pp. 289-302.

FAO (2006), Guidelines for soil profile description, $4^{\text {th }}$ edition, Food and Agriculture Organization of the
United Nations. Rome, Italy.

Hesse, P. R. (1998), A Textbook of soil chemical analysis, CBS Publishers \& Distributors, Delhi, India.

Ismail, H. A., Morsy, I., El-Zahaby, E. M. and El-Nagar, F. S. (2001), "A developed expert system for land use planning by coupling land information system and modeling", Alexandria Journal of Agricultural Research, Vol. 46 No. 3, pp. 141154.

Ismail, H. and Morsi, I. (2001), Applied System of Land Evaluation (ASLE) in arid zones (software), Soil and Water Science Department, Faculty of Agriculture, Alexandria University, Egypt.

ITT (2017), ITT Corporation, ENVI 5.1 Software, 1133 Westchester Avenue, White Plains, NY 10604, USA.

Jackson, M. L. (1973), Soil chemical analysis, Prentice-Hall of India, New Delhi.

Klute, A. and Dirksen C. (1986), Hydraulic conductivity and diffusivity: laboratory methods, In: Klute, A. (ed.), Methods of Soil Analysis, Part 1., 2nd ed. ASA Publications, Madison, WI., USA, pp. 687-734.

Marei, S. M., Ismail, H. A. and Meshref, H. (1987), "A computer program for land evaluation in Egypt", Mansoura University Journal of Agricultural Sciences, Vol. 12 No. 4 pp. $1427-$ 
1447.

Mclean, E. O. (1982), "Soil pH and lime requirement", In Page, A.L., Miller, R.H. and Keeney, D.R. (eds.,) Methods of soil analysis, Part 2 Chemical and Microbiological Properties, $2^{\text {nd }}$ edition. Agronomy 9: Am. Soil Sci. Soc., Madison, WI., USA, pp. 199-223.

Mevlut, U. (2016), "Determination of agricultural soil index using geostatistical analysis and GIS on land consolidation projects: A case study in Konya/Turkey", Computers and Electronics in Agriculture, Vol. 123, pp. 402-409.

Nelson, R. E. (1982), "Carbonate and gypsum", In: Page, A.L., Miller, R. H. and Keeney, D.R. (eds.), Methods of soil analysis, Part 2-Chemical and Microbiological properties, $2^{\text {nd }}$ ed., Agronomy 9, Soil Science Society of America, Madison, WI., USA, pp. 181-198.

O’Geen, A. T. (2008), Revised Storie Index for Use with Digital Soils Information, Water: 11, Publication 8335, Division of Agriculture and Natural Resources, University of California, USA.

Olsen S. R. and Sommers, L. E. (1982), Methods of Soil Analysis Part 2: Chemical and Microbiological Properties, Second ed., In: Page, A.L., Miller, R.H. and Keeney, D.R. (eds), American Society Agronomy, Madison, Wisconsin, USA, pp. 403430
Piper, C. S. (1950), Soil and plants analysis, InterScience, Inc., New York. USA.

Soil Survey Staff (1993), Soil Survey Manual, Soil Conservation Service, U.S. Department of Agriculture, Handbook, 18.

Sys, C. (1979), Evaluation of the physical environment for irrigation in terms of land characteristics and land qualities, World Soil Resources Reports (FAO), No. 50.

Sys, C. and Verheye, W. (1978), An Attempt to the Evaluation of Physical Land Characteristics for Irrigation According to the FAO Framework for Land Evaluation, International Training Center for Post Graduate Soil Scientists, Chent, Belgium, pp. 66-78.

Sys, C. E., Van, R. E., Debaveye, J. and Beernaert, F. (1993), Land evaluation, part III: crop requirements, General administration for development cooperation, Agricultural Publication, No. 7, Brussels, Belgiem, pp. 191-195.

Webster, R. and Oliver, M. A. (2007), Geostatistics for Environmental Scientists, Second edition, John Wiley \& Sons, West Sussex, England.

Zamil, B. A., Abd Allah, M. A., ElSalam, G. M. and El-Shahawy, M. I. (2009), "Land evaluation of old irrigated soils in North Delta region 
(Rewena Canal Area) at Kafr El Sheikh governorate", Alexandria Science Exchange Journal, Vol. 30

No. 2, pp. 206-227. 